\title{
Comparison of calcaneus joint internal and external fractures in open surgery and minimal invasive methods in patients
}

\author{
Alireza Amani (1), Vahid Shakeri (1), Alireza Kamali (2) \\ (1) Department of Orthopedic, Arak University of Medical Sciences, Arak, Iran \\ 2. Department of Anesthesiology, Arak University of Medical Sciences, Arak, Iran
}

This article is distributed under the terms of the Creative Commons Attribution Noncommercial License (CC BY-NC 4.0) which permits any noncommercial use, distribution, and reproduction in any medium, provided the original author(s) and source are credited.

\begin{abstract}
Surgical management of calcaneal fractures has various complications. In recent years, minimally invasive surgeries have been utilized for a wide range of surgical procedures on different body parts significantly declining complications. This paper compares surgical outcomes of calcaneal fracture management between the open reduction and internal fixation technique (ORIF) and the MIS technique. In this randomized clinical trial forty patients with calcaneus fractures were randomly assigned to two equal groups; ORIF group and MIS group. Patients were followed for 1 year post-operatively. Gissane and Bohler's angles, AOFAS questionnaire, pain intensity, ability to conduct previous activities and various complications like wound healing complications and irritation with shoe wear were assessed in the final postoperative visit. Eventually data obtained from the two groups were compared. All patients obtained fracture union. Bohler's and Gissane angles significantly increased and decreased, respectively, after the operation in both groups $(\mathrm{p}<0.05)$, however, no significant difference was found between the two research groups. AOFAS scores and pain intensity was similar in both groups, however, surgical duration in the MIS group was significantly less than the ORIF group $(\mathrm{P}=0.021)$. Only one patient from the ORIF group was not able to resume his previous occupation. 12 patients from the ORIF group and five patients from the MIS group experienced irritation from shoe wear $(p=0.025)$. In the ORIF group, 4 patients suffered from wound healing complications, however, none of the patients of the MIS group had wound complications $(\mathrm{p}=0.035)$. In conclusion, based on the results, it can be concluded that the MIS technique demonstrated better functional and radiographic outcomes as well as a more favorable complication profile, thus, it is recommended for the management of calcaneus fractures.
\end{abstract}

Key Words: Calcaneus internal and external fractures, open surgery, minimal invasive method Eur J Transl Myol 28 (2): 203-209, 2018

Calcaneus fracture $(\mathrm{CF})$ is the most common type of tarsal area fracture. ${ }^{1}$ These damages account for $2 \%$ of all fractures, ${ }^{2,3}$ and as much as $60 \%$ of tarsal fractures. ${ }^{4}$ Although there is no clear statistics of this fracture in Iran, global figures give us a good view of this issue. The reports indicate that as many as 2721 cases of CF were recorded in UK in 2010, while this number in the same year in the US was $17274 .^{5,6}$ It is estimated that $\mathrm{CF}$ is developing much faster among those living in developing countries. ${ }^{7,8}$ Although some calcaneus fractures can be viewed as minor damages, severe and serious damages are reported in most cases caused by high energy trauma. ${ }^{9}$ These damages are more common among the active youth and may have serious negative consequences for their daily activities and life quality. ${ }^{10,11}$ The clinical and functional results of $\mathrm{CF}$ treatment are usually far from being favorable and the damages caused to the soft tissue covering bones coupled with various fracture patterns make the treatment very difficult. ${ }^{11,12,13}$ What's more, a relatively long rehabilitation period follows the operation which may impair patient's performance. ${ }^{13,14}$ Due to the anatomical and biomechanical complications of foot and Thulacalcanal joint, any damage caused to this region will turn into a great challenge for orthopedic surgeons. Favorable reduction and preserving joint's congruity without causing serious damages to other hindfoot structures will be impossible. Improper treatment of 
these fractures will cause serious problems to gait (particularly while walking on uneven surfaces), pain, chronic joint stiffness, and subtalar and calcaneocuboid joints arthrosis ${ }^{12-14}$ Calcaneus widening would make it really difficult to put on normal shoes and a serious cosmetic problem may be caused among ladies ${ }^{12,15}$ Further to these problems, calcaneus fractures are also associated with significant economic consequences. Buckley et al showed that $20 \%$ of these people can not go back to their previous jobs even one year after the damage $^{10,11,16}$ Various therapeutic methods have been proposed for CF. Surgical treatments have been shown to be associated with more favorable consequences that non-surgical methods ${ }^{10,13,15,17,18}$ In type IV fractures based upon Sander's classification, early Arthrodesis is recommended. ${ }^{19,20}$ Open reduction internal fixation (ORIF) is the most common surgical method used to treat calcaneus fractures conducted with a lateral approach. In spite of its favorable consequences, there is great concern about the complications following soft tissue operation such as infection and wound dehiscence. Wound dehiscence have been reported among 17 to 20 percent of patients, ${ }^{21-24}$ and the frequency rate of $2.5 \%$ has also been reported for Osteomyelitis. ${ }^{25-28}$ To reduce the surgical complications, various percutaneous and minimally invasive methods have been proposed to treat $\mathrm{CF}$ which yielded favorable results. Various studies have pointed to the fact that such methods are associated with the considerable reduction in the post-calcaneus operation. ${ }^{4,29-31}$ These methods are based upon causing less damage to the soft tissue and achieving more favorable results and less complications. Of course, these methods are not without their problems. Compared to open surgery, achieving anatomical reduction and maintaining it is much more difficult and this problem shows itself more prominently in percutaneous methods. As a result, finding a method which can yield more favorable results in spite of these limitations is very important. ${ }^{27,31-33}$ The present research has sought to compare the clinical and functional outcomes and the occurrence rate of CF treatment in ORIF and MIS methods. The MIS technique utilized included the initial posterior facet placement using CARM guided pin followed by filling the empty space of Cancellous tissue in the lower surface of the facet using Bone Substitute (similar to Ballon Kyphoplasty technique) in order to prevent depression of joint surface.

\section{Materials and Methods}

This is a randomized clinical trial (RCT). Patients with calcaneus fracture resorting to Vali Asr Hospital of Arak who were qualified for inclusion were studied.

\section{Inclusion criteria}

Type II and III fractures based upon Sanders classification.

\section{Exclusion criteria}

Further fractures particularly on the same side, bilateral calcaneus fracture, multiple injury, open fracture, diabetes, Rheumatoid Arthritis, aging less than 15 years and fractures dating back to more than three weeks earlier. Based upon previous studies, the sample size in each group was set to at least 20 patients. The demographic and primary information including age, gender, length between fracture to operation, history of smoking cigarettes, and background diseases were all recorded.

\section{Clinica Imaging}

Ankle radiography was conducted in mortise, anteroposterior, lateral, Axial Harris View and lateral hind Foot angles. The Bohler and Gissen angles in the engaged side were also recorded. Lateral side radiography was conducted to measure the angles on the healthy side.

\section{Randomization}

Using the randomized numbers table, the patients were placed in two groups.

\section{Surgery}

Minimally invasive surgery group (MIS): the night following the operation, prep was performed for all patients in sterilized champ emphasizing the space between toes. The surgery was conducted under general or spinal anesthesia after receiving a dose of prophylactic antibiotics and closing the tourniquet in lateral position. The front lower limbs were placed on fluoroscopy bed in a scissor-like configuration. To prevent possible transmission of infection from the space between toes, the anterior part of the foot was separated from the field of operation using a rubber band or a size 8 glove. To reconstruct the height of calcaneus bone, size 3 or 4 pin on the external part of calcaneus was used to put pressure on medial from lateral. As a matter of fact, pressure was put towards the lower part of the posterior facet from the Tuberosity area of calcaneus under the control of a C-ARM. Next, the fracture was placed and Bohler and Gissane angles were recovered as much as possible. A 1 to $1.5 \mathrm{~cm}$ incision is made in $1.5 \mathrm{~cm}$ distal and $1 \mathrm{~cm}$ posterior distance to the tip of external malleolus so that the external wall of calcaneus can be observed. If the lateral wall in that region has no fractures, a valve with the same size will be made with osteotome so that we can enter the space between bones. If the posterior faust and the subtalar joint required further placement, the full placement would be conducted through the same space with pin and curt controlled by C-ARM. Then, 5 to 10 cc bone chips were put inside the bone and pressed towards the lower part of posterior faust. In most cases, we can continue the previous pin to calcaneocuboid 
joint and stabilize it in the same place as an auxiliary scaffold to preserve the reduction. Finally, reduction status was controlled using C-ARM. After rinsing, the wound was sutured and Jones bandage was performed. After opening the tourniquet, the foot was placed inside a short splint. The pin was removed 4 weeks later and partial weight bearing was allowed. The full weight bearing was allowed by the end of the second month. Open reduction internal fixation (ORIF): an L-shaped incision was made in the lateral surface of calcaneus. Peroneal tendons and Calcaneofibular ligament were retracted so that the fractures could be observed. The posterior faust was elevated and joint reduction became directly visible. The fracture was primarily fixed using $\mathrm{K}$-wire, then screw and plaque were utilized for final fixation. In cases where done defect was considerable, iliac bone graft was used to fill the defect. Following their discharge, the patients resorted on the second, fourth weeks and then on a monthly basis until union was observed based upon radiography and examination. By the end of the fourth month following the operation, CT scan was conducted to accurately study the subtalar joint and final union of parts. All patients were asked to resort to the hospital again one year following the operation. In the final visit, Bohler and Gissane angles were measured once again. AOFAS questionnaire was completed for all patients in order to examine the results of treatment. Visual analogue scale (VAS) was used to measure pain level. Patients were asked questions about their return to their previous jobs.

\section{Statistical Analyses}

Ultimately, the resulting data were statistically analyzed using SPSS v. 19 and Kolmogorov-Smirnov test was used to test data distribution normality. Wilcoxon test or Paired t-test were used to compare the data before and after treatment.

\section{Results and Discussion}

As many as 40 patients forming two groups each consisting of 20 participants were studied. The results of comparing background and demographic information are presented in Table 1. No significant differences were observed between the two groups. Radiographic assessments also showed that Bohler and Gissane angles on the healthy side were statistically similar in both groups (Table 1). Union was achieved in all patients. A comparison of pre-operation and post-operation values of these angles showed a significant increase in Bohler's angle and a significant decrease in Gissane angle ( $\mathrm{P}<$ $0.05)$. The differences between the two groups were not statistically significant (Tables 2, 3). A comparison of Bohler and Gissane angles one year following operation with the values correlated with healthy side angles showed that although surgery had helped to improve radiological outcomes in both groups, the operated foot in both groups exhibited a significant difference in its angle. We compared the Bohler and Gissane angle changes in the operated and healthy sides. It is clear that the changes in both groups are statistically the same (Table 4, 5). Table 6 presents the results of AOFAS score comparison, pain scale and the length of operation. The AOFAS score and the pain scale of the patients in both groups are similar but the length of operation in MIS group was significantly less than ORIF group $(\mathrm{P}=0.021)$. With the exception of only one patients in ORIF group, all patients regained the ability to resume their previous job and activities. As expected, 9 patients in ORIF group and 7 in MIS group were complaining of pain in heavy activities (VAS ranging from 3 to 6) and required painkillers for their pain, but the difference between the two groups was not significant $(\mathrm{P}=0.519) .12$ patients in ORIF and 5 in MIS group had difficulty putting on shoes. The difference between the two groups in this respect was significant $(\mathrm{P}=0.025)$. No cases of ulcer complications were observed in MIS group, while 2 cases of wound dehiscence, one case of surface infection and one case of deep infection were reported in ORIF group. Patients suffering from deep infection were treated using rinsing and debridement and venous antibiotics. The difference between the two groups was significant $(P=0.035)$. The most important finding of the present research was that in treating calcaneus fractures, the outcomes of MIS operation can be compared to those achieved through ORIF method with the clinically relevant difference of

Table 1. Demographic and background information

\begin{tabular}{|c|c|c|c|c|}
\hline \multicolumn{2}{|c|}{ Group } & Open $(n=20)$ & $\operatorname{MIS}(n=20)$ & P-value \\
\hline \multicolumn{2}{|c|}{ Age (years) } & $38.11 \pm 5.7$ & $35.11 \pm 7.6$ & 0.452 \\
\hline \multirow[t]{2}{*}{ Gender } & Male & 16 & 15 & \multirow[t]{2}{*}{0.705} \\
\hline & Female & 4 & 5 & \\
\hline \multirow[t]{2}{*}{ Fracture type } & Extra-articular & 3 & 6 & \multirow[t]{2}{*}{0.256} \\
\hline & Intra-articular & 17 & 14 & \\
\hline \multirow{2}{*}{$\begin{array}{c}\text { Sander's } \\
\text { classification }\end{array}$} & Type II & 8 & 11 & \multirow[t]{2}{*}{0.342} \\
\hline & Type III & 12 & 9 & \\
\hline \multicolumn{2}{|c|}{ Bohler's angle on the healthy side } & $28.2 \pm 6.6$ & $27.2 \pm 5.1$ & 0.157 \\
\hline \multicolumn{2}{|c|}{ Gissane angle on the healthy side } & $119.4 \pm 7.3$ & $116.5 \pm 9.2$ & 0.091 \\
\hline
\end{tabular}


Table 2. Bohler's angle before and after operation in each group and between groups

\begin{tabular}{|c|c|c|c|}
\hline Group & Open $(\mathrm{n}=20)$ & MIS (n=20) & P-value \\
\hline Before operation & $3 \pm 9.5$ & $7.3 \pm 6.1$ & 0.193 \\
\hline $\begin{array}{c}\text { One year following the } \\
\text { operation }\end{array}$ & $25.2 \pm 7.3$ & $24.3 \pm 3.3$ & 0.121 \\
\hline P-value & $<0.001$ & $<0.001$ & \\
\hline
\end{tabular}

Table 3. Gissane angle before and after operation in each group and between groups

\begin{tabular}{|c|c|c|c|}
\hline Group & Open $(\mathrm{n}=20)$ & MIS $(\mathrm{n}=20)$ & P-value \\
\hline Before operation & $141.3 \pm 2.9$ & $142.3 \pm 9.6$ & 0.165 \\
\hline $\begin{array}{c}\text { One year following the } \\
\text { operation }\end{array}$ & $123.4 \pm 1.3$ & $121.4 \pm 5.1$ & 0.277 \\
\hline P-value & $<0.001$ & $<0.001$ & \\
\hline
\end{tabular}

Table 4. Bohler's angle in the healthy and damaged sides in the final visit

\begin{tabular}{|c|c|c|c|}
\hline Group & Open $(\mathrm{n}=20)$ & MIS $(\mathrm{n}=20)$ & P-value \\
\hline Healthy side & $28.2 \pm 6.6$ & $27.2 \pm 5.1$ & \\
\hline Damaged side & $25.2 \pm 7.3$ & $24.3 \pm 3.3$ & \\
\hline P-value & 0.002 & 0.001 & 0.968 \\
\hline Difference between sides & $2.3 \pm 9$ & $3.4 \pm 2$ & \\
\hline
\end{tabular}

Table 5. Gissane angle in the healthy and damaged sides in the final visit

\begin{tabular}{|c|c|c|c|}
\hline Group & Open $(\mathrm{n}=20)$ & MIS $(\mathrm{n}=20)$ & P-value \\
\hline Healthy side & $119.4 \pm 7.3$ & $116.5 \pm 9.2$ & \\
\hline Damaged side & $123.4 \pm 1.3$ & $121.4 \pm 5.1$ & 0.383 \\
\hline P-value & 0.028 & 0.003 & \\
\hline Difference between sides & $3.1 \pm 5.3$ & $4.3 \pm 6$ & \\
\hline
\end{tabular}

less risk of complications in patients treated with MIS technique. Although surgical treatments yield good results, the type and occurrence rata of post-operation complications is a great concern of surgeons. Various problems may prolong treatment and hospitalization periods and impose considerable physical and spiritual costs to the patient, his family and treatment team. Finding the ways to reduce these complications is a great concern for many researchers. The idea of minimally invasive and percutaneous operations has recently been proposed and a lot of attention has been paid to that. Calcaneus fracture has always been a great challenge due to many reasons. Recovering the joint surface in some fractures can be really difficult leading to further complications. What's more, the occurrence rate of ulcer complications and infection among these patients is a major concern. ${ }^{21,22,25,34}$ As a result, several minimally invasive and percutaneous methods were introduced and used for the managements of this fractures. ${ }^{4,27,33,34}$ Considering the results achieved in many researches, using them to treat calcaneus fractures sounds rational and appropriate as these techniques seek to minimize soft tissue iatrogenic injuries and disruptions of blood circulation in bones. As the results of previous researches can be really paradoxical and, even, different and they can't be used to decide about using or not using minimally invasive techniques to treat calcaneus fracture, we decided to make a

Table 6. AOFAS score, pain scale, and the length of operation across the two groups

\begin{tabular}{|c|c|c|c|}
\hline Group & Open $(\mathrm{n}=20)$ & MIS $(\mathrm{n}=20)$ & P-value \\
\hline AOFAS & $77.10 \pm 4.2$ & $79.13 \pm 6.3$ & 0.112 \\
\hline Pain scale & $2.1 \pm 6.7$ & $2.1 \pm 2.4$ & 0.362 \\
\hline Length of operation & $72.8 \pm 3.3$ & $65.6 \pm 6$ & 0.021 \\
\hline
\end{tabular}


comparison between these two methods in the present research. Two relatively similar groups of patients were studied in our research. As the results indicate, the important angles indicating calcaneus axis (Gissane and Bohler's) were favorably recovered. Of course, the final angles in both groups exhibited a significant difference with what was observed on the healthy side. Although this difference was not numerically significant, the complicated biomechanics of foot and ankle show that even these limited values may make the individual prone to further and long-term problems such as heel pain. The results achieved in our research also showed that a considerable percentage of patients felt pain in heavy physical activities. Although the bone is exposed in ORIF and direct observation plays a major role in increasing the capability of operation in recovering the joint surface and in improving calcaneus axis, MIS technique also yielded results similar to those achieved by ORIF. This finding points to the fact that MIS is a very good and effective technique compared to ORIF. The performance results in terms of AOFAS, pain scale, and the rate of resuming previous activities were similar in all groups which can be another confirmation of our claim. As far as we are concerned, few researches have sought to compare ORIF and minimally invasive methods in treating calcaneus fractures. The importance of minimally invasive methods in reducing the risk of complications such as wound recovery issues, and surgical infections was also highlighted. In the present research, the occurrence rate of complications such as infection, dehiscence and the problems associated with putting on shoes in the MIS group was significantly less than what was observed in ORIF group. Considering the fact that these two complications are the most common therapeutic problems in surgical treatment of calcaneus fractures, this results can be really inspiring and favorable. Few comparative studies have been conducted on treating calcaneus fractures using ORIF and MIS methods (various techniques) many of which are retrospective. In one of these studies, Kline et al conducted a retrospective research to compare the results of treating the fractures inside calcaneus joint using ORIF and MIS methods. Similar to the present research, the researchers concluded that the clinical results were similar across the two groups, but the ulcer's complication and need for secondary operations in MIS group was significantly less. ${ }^{35}$ In another retrospective research, $\mathrm{Wu}$ et al realized that ulcer's complications $(1.9 \%$ vs. $11.8 \%)$ and the time when weight bearing began (5.6 vs. 9.4 days) in MIS group were significantly less, while AOFAS in both groups were the same. According to Wu et al., MIS reduces complications, shortens the weight bearing time and is as effective as ORIF. ${ }^{34}$ Also Yeo et al compared the results of treating fractures inside calcaneus joint through ORIF with lateral extensile approach and sinus tarsi approach (mini incision). The researchers arrived at the conclusion that no significant difference can be observed between the two groups in terms of the clinical and radiological outcomes, but significantly greater levels of ulcer complications were reported in lateral extensile approach. ${ }^{36}$ On the other hand, other researches have not confirmed the reduction of complications using MIS methods. For instance, Takasaka et al compared three ORIF methods using plaque, external fixation, and MIS using nuts and wire arriving at the conclusion that there are no differences between these three methods. ${ }^{37}$

On the other hand, we should keep in mind that none of these studies have utilized the reduction methods described in the present research and they can't be used properly in studying the results of the present research. Feng et al., 2017 compared the results of treating fractures inside the calcaneus joint through MIS method using sinus tarsi approach utilizing plaque and fixation with percutaneous screwing along with calcium phosphate cement. These researchers finally arrived at the conclusion that both MIS methods were associated with comparable results, although the time of operation and the complications of the ulcer in screwing group was less and the subtalar joint exhibited more motion. On the other hand, in sinus tarsi group, calcaneus width was recovered better and more accurate reduction of the joint surface was possible. ${ }^{38}$ In conclusion, our results show that treating calcaneus fractures following MIS method using percutaneous screwing and cement injection may yield functional, clinical and radiographic results comparable to ORIF method, but the occurrence of ulcer complications and of the problems associated to shoes decreased significantly.

\section{List of acronyms}

AOFAS -American orthopaedic foot and ankle society

$\mathrm{C}$-arm - is a term for a scanner imaging intensifier

$\mathrm{CF}$ - calcaneus fracture

ORIF - open reduction internal fixation

MIS - minimally invasive surgery

RCT - randomized clinical trial

VAS - visual analogue scale

\section{Author's contributions}

Authors equally contributed to the manuscript.

\section{Acknowledgments}

We are thankful to the personals of operating room and orthopedic ward of Arak Valiasr Hospital.

\section{Fundings}

None

\section{Conflict of Interest}

The authors report no conflicts of interests.

\section{Ethical Publication Statement}

We confirm that we have read the Journal's position on issues involved in ethical publication and affirm that 
this report is consistent with those guidelines.

\section{Corresponding Author}

Alireza Kamali, Department of Anesthesiology, Arak University of Medical Sciences, Arak, Iran

Phone: +989181622810

Email: Dr.kamali@arakmu.ac.ir

\section{E-mails of co-authors}

Alireza Amani: dramani.ortho@yahoo.com Vahid Shakeri: dr.shakerivahid@yahoo.com

\section{References}

1. Biggi F, Di Fabio S, D'Antimo C. Percutaneous calcaneoplasty in displaced intraarticular calcaneal fractures. J Orthop Traumatol 2013;14:307-10.

2. Mitchell MJ, McKinley JC, Robinson CM. The epidemiology of calcaneal fractures. Foot (Edinb) 2009;19:197-200.

3. Schepers T, van Lieshout EM, van Ginhoven TM, et al. Current concepts in the treatment of intraarticular calcaneal fractures: results of a nationwide survey. Int Orthop 2008;32:711-5.

4. Ene R, Popescu D, Panaitescu C, et al. Low complications after minimally invasive fixation of calcaneus fracture. J Med Life 2013;6:80-3.

5. NHS Information Centre. Hospital episode statistics: 2000-2012. www.hesonline.nhs.uk.

6. Agency for Healthcare Research and Quality. HCUP nationwide inpatient sample. 2010. www.hcup-us.ahrq.gov/nisoverview.jsp.

7. Dhillon MS, Aggarwal S, Dhatt S, Jain M. Epidemiological pattern of foot injuries in India: preliminary assessment of data from a tertiary hospital. J Postgrad Med Edu Res 2012;46:144-7.

8. Tadros AM, Eid HO, Abu-Zidan FM. Epidemiology of foot injury in a high-income developing country. Injury 2010;41:137-40.

9. Griffin D, Parsons N, Shaw E, et al. UK Heel Fracture Trial Investigators. Operative versus nonoperative treatment for closed, displaced, intraarticular fractures of the calcaneus: randomised controlled trial. BMJ 2014;349:g4483.

10. Buckley R, Tough S, McCormack R, et al. Operative compared with nonoperative treatment of displaced intraarticular calcaneal fractures: a prospective, randomized, controlled multicenter trial. J Bone Joint Surg Am 2002;84-A(10):173344.

11. Pozo JLL, Kirwan EO, Jackson AM. The longterm results of conservative management of severely displaced fractures of the calcaneus. J Bone Joint Surg Br 1984;66:386-90.

12. Crosby LA, Fitzgibbons T. Intraarticular calcaneal fractures. Results of closed treatment. Clin Orthop Relat Res 1993;290:47-54.

13. Su Y, Chen W, Zhang T, et al. Bohler's angle's role in assessing the injury severity and functional outcome of internal fixation for displaced intraarticular calcaneal fractures: a retrospective study. BMC Surg 2013;13:40.

14. Clare MP, Crawford WS. Managing complications of calcaneus fractures. Foot Ankle Clin N Am 2017;22:105-16.

15. Meena S, Gangary SK, Sharma P. Review Article: Operative versus nonoperative treatment for displaced intraarticular calcanealfracture: a metaanalysis of randomised controlled trials. J Orthop Surg (Hong Kong) 2016;24(3):411-6.

16. Luo X, Li Q, He S, He S. Operative Versus Nonoperative Treatment for Displaced IntraArticular Calcaneal Fractures: A Meta-Analysis of Randomized Controlled Trials. J Foot Ankle Surg 2016;55:821-8.

17. Zhang W, Lin F, Chen E, et al. Operative Versus Nonoperative Treatment of Displaced IntraArticular Calcaneal Fractures: A Meta-Analysis of Randomized Controlled Trials. J Orthop Trauma 2016;30:e75-81.

18. Agren PH, Wretenberg P, Sayed-Noor AS. Operative versus nonoperative treatment of displaced intra-articular calcaneal fractures: a prospective, randomized, controlled multicenter trial. J Bone Joint Surg Am 2013;95:1351-7.

19. López-Oliva F, Sánchez-Lorente T, Fuentes-Sanz A, et al. Primary fusion in worker's compensation intraarticular calcaneus fracture. Prospective study of 169 consecutive cases. Injury 2012;43 Suppl 2:S73-8.

20. Holm JL, Laxson SE, Schuberth JM. Primary subtalar joint arthrodesis for comminuted fractures of the calcaneus. J Foot Ankle Surg 2015;54:61-5.

21. Al-Mudhaffar M, Prasad CV, Mofidi A. Wound complications following operative fixation of calcaneal fractures. Injury 2000;31:461-4.

22. Assous M, Bhamra MS. Should Os calcis fractures in smokers be fixed? A review of 40 patients. Injury 2001;32:631-2.

23. Folk JW, Starr AJ, Early JS. Early wound complications of operative treatment of calcaneus fractures: analysis of 190 fractures. J Orthop Trauma 1999;13:369-72.

24. Harvey EJ, Grujic L, Early JS, et al. Morbidity associated with ORIF of intra-articular calcaneus fractures using a lateral approach. Foot Ankle Int 2001;22:868-73.

25. Shuler FD, Conti SF, Gruen GS, Abidi NA. Wound-healing risk factors after open reduction and internal fixation of calcaneal fractures: does correction of Bohler's angle alter outcomes? Orthop Clin North Am 2001;32:187-92.

26. Brauer CA, Manns BJ, Ko M, et al. An economic evaluation of operative compared with nonoperative management of displaced intraarticular calcaneal fractures. J Bone Joint Surg Am 2005;87:2741-9. 


\section{Open surgery vs minimal invasive methods for calcaneus fractures}

Eur J Transl Myol 28 (2): 203-209, 2018

27. Abdelazeem A, Khedr A, Abousayed M, et al. Management of displaced intra-articular calcaneal fractures using the limited open sinus tarsi approach and fixation by screws only technique. Int Orthop 2014;38:601-6.

28. Arastu M, Sheehan B, Buckley R. Minimally invasive reduction and fixation of displaced calcaneal fractures: surgical technique and radiographic analysis. Int Orthop 2014;38:539-45.

29. Labbe JL, Peres O, Leclair O, et al. Minimally invasive treatment of displaced intra-articular calcaneal fractures using the balloon kyphoplasty technique: preliminary study. Orthop Traumatol Surg Res 2013;99:829-36.

30. Su Y, Chen W, Zhang Q, et al.. Bony destructive injuries of the calcaneus: long-term results of a minimally invasive procedure followed by early functional exercise: a retrospective study. BMC Surg. 2014;14:19.

31. Xia S, Wang X, Lu Y, et al. A minimally invasive sinus tarsi approach with percutaneous plate and screw fixation for intra-articular calcaneal fractures.Int J Surg 2013;11:1087-91.

32. Stulik J, Stehlik J, Rysavy M, Wozniak A. Minimally-invasive treatment of intra-articular fractures of the calcaneum. J Bone Joint Surg Br 2006;88:1634-41.

33. Gomaa MA, El Naggar A, Anbar AS. A new minimally invasive technique for the treatment of intraarticular fractures of the calcaneus, preliminary results. Egyptian Orthopedic Journal 2014;49:225-30.
34. Wu Z, Su Y, Chen W, et al. Functional outcome of displaced intra-articular calcaneal fractures: a comparison between open reduction/internal fixation and a minimally invasive approach featured an anatomical plate and compression bolts. J Trauma Acute Care Surg 2012;73:743-51.

35. Kline AJ, Anderson RB, Davis WH, et al. Minimally invasive technique versus an extensile lateral approach for intra-articular calcaneal fractures. Foot Ankle Int 2013;34:773-80.

36. Yeo J-H, Cho H-J, Lee K-B. Comparison of two surgical approaches for displaced intra-articular calcaneal fractures: sinus tarsi versus extensile lateral approach. BMC Musculoskeletal Disorders 2015;16:63.

37. Takasaka M, Bittar CK, Mennucci FS, et al. Comparative study on three surgical techniques for intra-articular calcaneal fractures: open reduction with internal fixation using a plate, external fixation and minimally invasive surgery. Rev Bras Ortop 2016;51:254-60

38. Feng Y, Shui X, Wang J, et al. Comparison of percutaneous cannulated screw fixation and calcium sulfate cement grafting versus minimally invasive sinus tarsi approach and plate fixation for displaced intra-articular calcaneal fractures: a prospective randomized controlled trial. BMC Musculoskeletal Disorders 2016;17:288.

Submission: $11 / 03 / 18$

Acceptance: 23/03/18 